\title{
Endocrinomic profile of neurointermediate lobe pituitary prohormone processing in PC1/3- and PC2-Null mice using SELDI-TOF mass spectrometry
}

\author{
Atira Hardiman ${ }^{1}$, Theodore C Friedman', William C Grunwald Jr' ${ }^{1}$, Machi Furuta ${ }^{3}$, \\ Ziaorong Zhu ${ }^{3}$, Donald F Steiner ${ }^{3}$ and David R Cool ${ }^{1}$ \\ ${ }^{1}$ Department of Pharmacology and Toxicology, School of Medicine, Wright State University, Dayton, Ohio 45435, USA \\ ${ }^{2}$ Division of Endocrinology, Metabolism and Molecular Medicine, Charles R Drew University, Los Angeles, California 90059, USA \\ ${ }^{3}$ Department of Biochemistry and Molecular Biology, University of Chicago, Chicago, Illinois 60637, USA \\ (Requests for offprints should be addressed to D R Cool; Email: david.cool@ wright.edu)
}

(M Furuta is now at The First Department of Medicine, Wakayama University of Medical Science, 811-1 Kimiidera, Wakayama 641-9509, Japan)

\begin{abstract}
Pro-vasopressin and pro-oxytocin are prohormones processed in the neurointermediate lobe pituitary to form the biologically active peptide hormones, arginine vasopressin (AVP) and oxytocin. Neurointermediate lobe pituitaries from normal $(+/+)$, heterozygous (+/-), PC2-Null (-/-), PC1/3-Null and oxytocin-Null mice were analyzed by SELDI-TOF mass spectroscopy for the peptide hormone products, AVP, oxytocin and neurophysin I and II. Molecular ion species with masses characteristic of oxytocin, AVP, neurophysin I and II, i.e. 1009.41, 1084.5, 9677 and 9679 daltons respectively, were identified in all but the oxytocin-Null mice by comparison with synthetic standards or by C-terminal sequence analysis. Other ion species were found specifically in PC2-Null, heterozygote or normal mice. The results indicate that, in mice, both $\mathrm{PC} 1 / 3$ or $\mathrm{PC} 2$ enzyme activity are capable, but not required to correctly process pro-vasopressin or pro-oxytocin to their constituent active peptide hormones.
\end{abstract}

Journal of Molecular Endocrinology (2005) 34, 739-751

\section{Introduction}

The investigation of the proteome is beginning to provide new and exciting insights in the field of endocrinology. To facilitate endocrine proteomic studies (endocrinomics), new and diverse methods for mass spectrometry have been developed that offer greater sensitivity and resolution to more accurately analyze and identify small peptides (van Strien et al. 1996, Jimenez et al. 1997, Slominski et al. 2000). One of the newer mass spectrometric techniques is surface enhanced laser/ desorption ionization-time of flight mass spectrometry or SELDI-TOF MS (Ciphergen, Palo Alto, CA, USA) (Hutchens \& Yip 1993). SELDI-TOF MS utilizes chemically treated metal chips, ProteinChips, that specifically bind peptides and proteins based on their chemical characteristics (Hutchens \& Yip 1993, Merchant \& Weinberger 2000). Following a wash step, peptides and proteins retained on the chips are analyzed by time of flight mass spectrometry. Thus, using SELDI-TOF MS, proteins and peptides in crude tissue extracts can be systematically and quantitatively analyzed at a greater resolution and specificity than by traditional techniques e.g. SDS-PAGE, Western blots or
RIA. By coupling SELDI-TOF MS techniques with analysis of endocrine tissues from genetically modified mice, prohormone processing and peptide hormone synthesis can be studied in greater detail than before.

Biologically active neuroendocrine peptide hormones are generated through the coordinated action of intragranular conditions and specific enzymes that cleave prohormones at paired-basic residues (Loh 1988, Darby \& Smyth 1990, Lindberg 1991, Seidah et al. 1992, Zhou et al. 1999). Two enzymes from the subtilisin-like enzyme family have been identified that are proposed to be involved in nearly all neuroendocrine prohormone processing, PG1/3, also known as SPC3, and PC2, also known as SPC2 (Steiner et al. 1992, Seidah et al. 1993). (At the 6th Gordon Research Conference on Proprotein Processing, Trafficking and Secretion (2004), the leading researchers agreed to use the terminology, PC1/3, to describe the identical PC1 and PC3 prohormone convertase.) The two prohormones found in the posterior lobe of the pituitary, pro-oxytocin and pro-vasopressin, were proposed, based on co-localization studies, to be processed to their biologically active peptide hormones, oxytocin and arginine vasopressin (AVP), by one or both of these enzymes (Dong et al. 1997). Previous in vitro 
studies have suggested that PG1/3 can cleave pro-vasopressin and pro-oxytocin to AVP and oxytocin respectively (Coates \& Birch 1998), while the processing of these prohormones by PC2 has not been examined. However, in humans, pro-vasopressin and pro-oxytocin processing is impaired when there is a putative deficit in processing by the PC2 enzyme, such as in patients with Prader-Willi syndrome or Wolfram's syndrome (Gabreels et al. 1994, 1998, Swaab et al. 1995).

In this study, genetically modified mice, deficient in the processing enzyme PG1/3 (Zhu et al. 2002b) or PC2 (Furuta et al. 1997), were analyzed by SELDI-TOF MS. Analyses of the neurointermediate lobe pituitaries from PC1/3-Null or PC2-Null mice indicate that the lack of each of these enzymes had no effect on the efficient processing of pro-vasopressin and pro-oxytocin, while the processing of other peptides was affected.

\section{Materials and methods}

\section{PC1/3-, PC2- and oxytocin-Null mice}

For the PG1/3-Null mice, a neomycin cassette was inserted in the first exon of the PC1/3 gene (Zhu et al. 2002b). For the PC2-Null mice, a neomycin cassette was inserted in the third exon of the PG2 gene (Furuta et al. 1997). Both lines of mice were bred to generate wild-type, heterozygote and null offspring. OxytocinNull mice (Nishimori et al. 1996) were generously provided by Dr Mariana Morris, Wright State University, Dayton.

\section{Ciphergen ProteinChip SELDI-TOF mass spectrometry}

For direct analysis of mouse neurointermediate lobe pituitary peptides, the pituitaries were homogenized in $0 \cdot 1 \mathrm{M} \mathrm{HCl}$ containing $1 \mathrm{mM}$ PMSF and the cell debris removed by centrifugation $(5 \mathrm{~min}$ at $15000 \times \boldsymbol{g})$. One microgram of each sample was spotted onto a weak cation exchange (WCX2) ProteinChip (Ciphergen) for $30 \mathrm{~min}$, washed with $5 \mu \mathrm{l}$ distilled water and allowed to dry. For comparison and quantitation, WCX2 ProteinChips were spotted with different concentrations of reconstituted oxytocin and AVP standards (Bachem, Torrance, CA, USA). Matrix, alpha-cyano-4-hydroxy cinnamic acid (CHCA) in 50\% acetonitrile containing $0 \cdot 1 \%$ trifluoroacetic acid (TFA) $(0.5 \mu \mathrm{l})$, was added to the spots and allowed to dry. The ProteinChips were analyzed on a Ciphergen SELDI Protein Biology System II (Ciphergen) with a spot protocol that ionized each spot four times over twenty different areas. The spot was initially ionized with1 laser hit at an intensity of 200, with the sensitivity set at 10 , followed by 4 laser hits at 195 intensity. The source voltage was set at 20000 volts and the detector voltage at 1900 volts. The 80 laser hits were averaged for each spot and the resulting protein profile analyzed to determine the mass of each peak. Ciphergen ProteinChip software was used to integrate the area under each peak for use in quantitative analysis. For the AVP and oxytocin standards, the integrated area under each peak was plotted against the corresponding concentration of peptide and the best-fit line was analyzed by linear regression.

\section{On-chip C-terminal peptide sequencing}

Neurointermediate lobe tissue lysates ( $1 \mu \mathrm{g}$ protein) were spotted on an H4 ProteinChip and air-dried, as previously described (Cool \& Hardiman 2004). The ProteinChip was placed in a 'moist chamber' - a pipette tip box (with lid) containing a wet paper towel. For the sequencing reaction, carboxypeptidase Y (CPY) (Pierce, Rockford, IL, USA) (30 units/ $\mu \mathrm{l})$ was diluted to $2 \cdot 4 \times 10^{-3}$ units $/ \mathrm{ml}$ in $50 \mathrm{mM}$ MES, $\mathrm{pH}$ 6.5. One microliter of each dilution was added to each spot on the ProteinChip, the lid closed and the chamber placed in a $37^{\circ} \mathrm{C}$ incubator for $5 \mathrm{~min}$. After incubation, $1 \mu \mathrm{l}$ of a supersaturated solution of matrix, CHCA in 50\% acetonitrile containing $0 \cdot 1 \%$ TFA, was added to each spot to stop the reaction. The ProteinChip was then analyzed in a Ciphergen PBSII ProteinChip reader.

\section{Results}

\section{Proteomic analysis of neurointermediate lobe pituitaries by SELDI-TOF MS}

SELDI-TOF MS analysis of the neurointermediate lobe pituitaries from the nine mice (PC2-Null, PC2heterozygote and PC2 wild-type) showed four distinct profiles between 1000 and 12000 daltons. In the first profile, the ion species between 1600 and 1750 daltons were identified as being present in the heterozygote and normal genotype, but absent from or greatly diminished in the PC2-Null mice ( $\mathrm{a}$ in Fig. 1). These peaks have previously been identified as des-acetyl- $\alpha$-melanocyte stimulating hormone (MSH), $\alpha$-MSH and di-acetyl- $\alpha-$ MSH ( 1620, 1660 and 1702 daltons respectively) (Cool \& Hardiman 2004). In the second region, ion species between 2350 and 2506 daltons were also identified in the heterozygote and normal genotype, but were absent from the PC2-Null genotype (b in Fig. 1). These ion peaks were identified by matrix-assisted laser/desorption ionization (MALDI)-TOF/TOF as the CLIP region of adrenocorticotropin (2505 daltons for CLIP and 2350 daltons after loss of the C-terminal Phe-residue) (S M Leung and D R Cool, unpublished data). In the third region, a series of ion peaks between 2700 and 3500 daltons, representing acetylated and non-acetylated forms of $\beta$-endorphin ${ }_{1-27}$ through $\beta$-endorphin ${ }_{1-31}$, were identified as different in the 


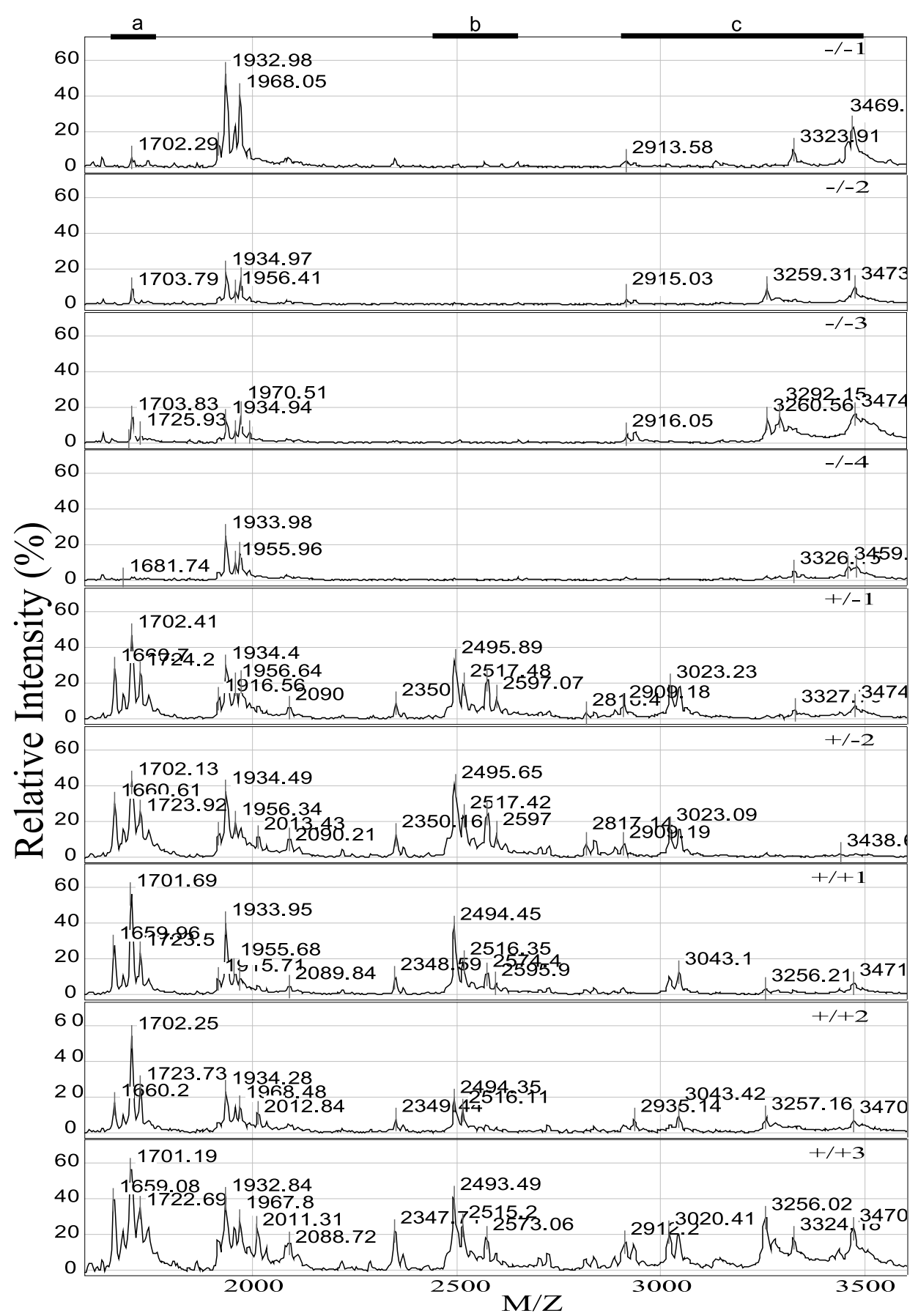

Figure 1 SELDI-TOF mass spectrometric profiles of neurointermediate lobe pituitaries from PC2-Null $(-/-)$, heterozygote $(+/-)$ and normal mice $(+/+)$. Neurointermediate lobe pituitary lysates were applied to each spot on a Ciphergen SELDI-TOF WCX2 ProteinChip. The ProteinChip was analyzed on a Ciphergen PBSII SELDI-TOF mass spectrometer using version 3.0 of the Ciphergen software. The relative intensity (\%) plotted for each peptide/protein (mass/charge, M/Z) is shown for the region between 1500 and 3600 daltons. Regions where peptides are noticeably changed in the PC2-Null mice compared with heterozygote and wild-type mice are indicated by a black bar with the letter $a, b$ or $c$ above. 
PC2-Null compared with heterozygote and normal mice (c in Fig. 1). The results clearly show differences in the proteomic profile between PC2-Null, heterozygote and normal mice. The differences between these spectra represent the first step in characterizing the effect of 'knocking out' a major processing enzyme in a mouse model.

\section{Analysis of oxytocin and AVP processing by SELDI-TOF MS}

Oxytocin and AVP are the two predominant peptide hormones present in the neurointermediate lobe pituitary. Fully processed and amidated AVP (1086.5 daltons and AVP+sodium, 1105.92 daltons) and oxytocin+sodium (1031.23 daltons) appeared in the spectra generated from all the PC2 mice (Fig. 2). A small amount of oxytocin (1009.22 daltons) was observed in only one PC2-Null mouse (Fig. 2). Subsequent analysis of other neurointermediate lobe pituitaries showed varying amounts of the 1009.22 dalton form of oxytocin. The predominant peak for oxytocin was the sodium peak at 1031 daltons. AVP and oxytocin were definitively identified by comparison with AVP and oxytocin synthetic standards analyzed by SELDI-TOF MS, as previously shown (Cool \& DeBrosse 2003). The identity of oxytocin was confirmed by the presence of ion peaks of the expected mass (1009.22 and 1031.23 dalton) in pituitaries of wild-type mice compared with the absence of these two peaks in spectra generated from a neurointermediate lobe of oxytocin-Null mice (OTNull) (Fig. 2). In addition, this spectrum was compared with spectra generated from PG1/3-Null mice (Fig. 3), where it was apparent that AVP and oxytocin were generated in the absence of $\mathrm{PC} 1 / 3$.

The relative amount of oxytocin and AVP in the samples was determined by integrating the corresponding ion peak areas (Table 1). There was no significant difference $(P>0.05)$ in the integrated area of the peaks for oxytocin or AVP (and their sodium adducts) between PC2-Null, heterozygote and normal mice (Table 1).

\section{Neurophysin I and II}

The peptides, oxytocin and AVP, are generated by cleaving pro-oxytocin and pro-vasopressin at the junction between oxytocin or AVP and their respective neurophysin regions (neurophysin I from pro-oxytocin and neurophysin II from pro-AVP), followed by removal of the basic residues and amidation. Therefore, the neurophysin regions of the two prohormones should also be present if oxytocin and AVP were correctly processed. When the profiles for each of the mice were analyzed, a series of ion peaks was found between 9000 and 10000 daltons (Fig. 4). The predicted masses for mouse neurophysin I and neurophysin II were determined using DNA Strider software (Marck 1988) and are shown in Tables 2 and 3 respectively. In the PC2-Null, heterozygote and wild-type mice, an ion peak at $\sim 9677$ daltons was identified that correlated with the predicted size of neurophysin I and II (Tables 2 and 3 respectively). This peak was not observed in tissues that do not make oxytocin or AVP, e.g. anterior pituitary (bottom plot in Fig. 4). Finally, an ion peak at 8150 daltons was identified as being specific to the PC2-Null genotype (Fig. 4). Antibody capture analysis using antibodies to neurophysin failed to identify this peak as part of the neurophysin molecule. Thus, this peak is not neurophysin related.

\section{On-chip peptide sequencing}

To more accurately determine the presence and identity of the neurophysin peptides, pituitary lysates were dried on Ciphergen hydrophobic H4 ProteinChips and incubated with CPY, a protease that systematically removes amino acids from the C-terminus of peptides and proteins. Preliminary analysis revealed ion peaks of sizes 9676, 9519, 9369, 9304, 9153 daltons (Fig. 5A and Tables 2 and 3). This multiple peak (or ragged end) pattern has been observed in neurointermediate lobe pituitaries from nearly 1000 mice (D R Cool, unpublished results) and represents the naturally occurring C-terminal amino acid(s) truncated state for neurophysin. Analysis of the ion peaks and the difference in their mass indicates an amino acid sequence that corresponded to the $\mathrm{C}$-terminal amino acid sequence for neurophysin I, FSER (Fig. 5B). Upon treatment with CPY, the major ion peak shifted, to generate an additional two amino acids, SAFSER (Fig. 5B, Table 2). The ion peak for neurophysin II was nearly identical in size to the predicted peak size for neurophysin I and could not be distinguished from neurophysin I (Fig. 5A). After CPY treatment of another sample, several peaks were identified (sizes 9585, 9473, 9314.8, 9166.2 daltons), yielding the amino acid sequence, FRLT, that corresponds to the last four $\mathrm{C}$-terminus amino acids of neurophysin II (Fig. 5C, Table 3). The ion peak at 9094 in Fig. 5C, was 8 daltons from the 9086 peak of neurophysin I, and thus could not be positively identified as coming from neurophysin I.

The oxytocin-Null mouse was expected to lack ion peaks for neurophysin I. When neurointermediate lobe pituitary tissue was analyzed by SELDI-TOF MS, two ion peaks, 9519 and 9389 daltons that correspond to neurophysin I, were missing (Fig. 5D). Three ion peaks remained, 9677, 9471 and 9315 daltons, that correspond to neurophysin II, providing further proof that this series of ion peaks represent the neurophysin peptides.

In addition to the neurophysin region, pro-vasopressin contains a 39 amino acid C-terminal region, the glycopeptide region. This glycopeptide is the only 


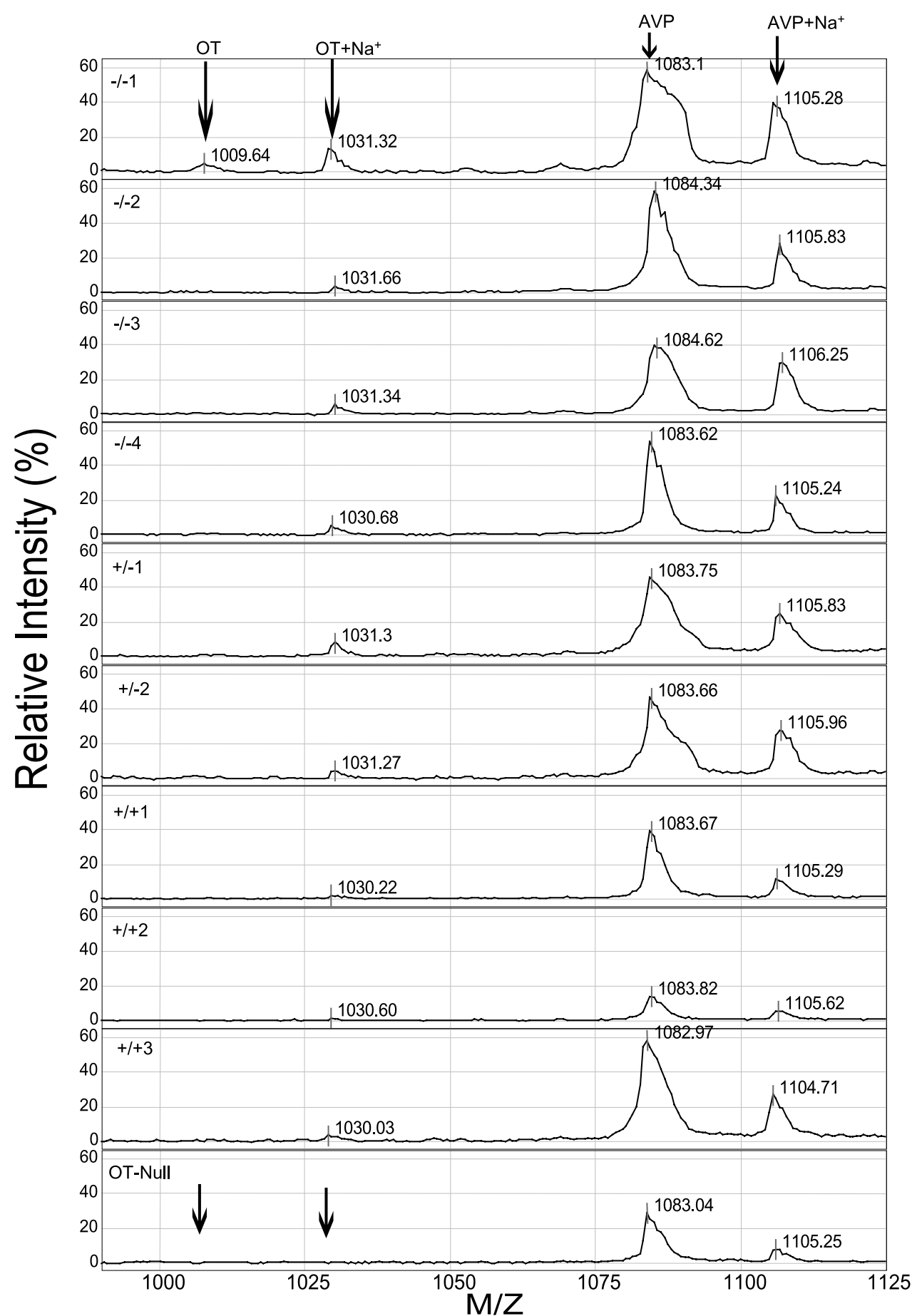

Figure 2 SELDI-TOF mass spectrometric profiles of oxytocin (OT) and AVP in PC2-Null (-/-), heterozygote $(-/+)$, wild-type $(+/+)$ mice or OT-Null mice. Oxytocin is seen as a small ion peak at 1009.64 daltons with a sodium ion at 1031.32 daltons in the first $-/$-spectra. Sodium adds approximately 22 daltons to the mass. The 1009.64 dalton ion is absent from the other animals. For AVP, a larger 1083.1 dalton ion was present in all samples with the sodium ion appearing at $1105 \cdot 28$ daltons. In the OT-Null profile, the arrows indicate the lack of an ion peak for oxytocin or its sodium salt. 


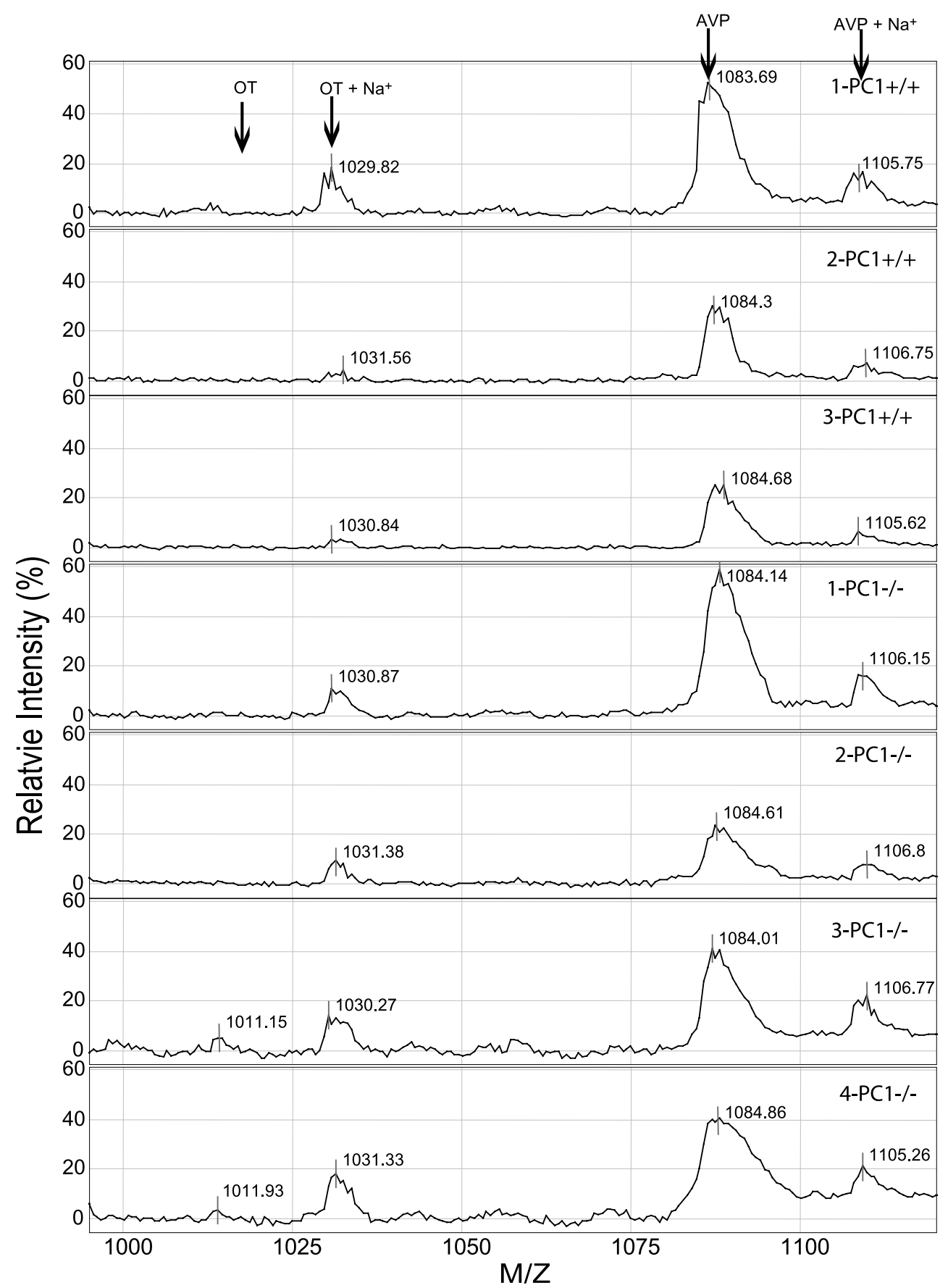

Figure 3 SELDI-TOF MS profile of oxytocin (OT) and AVP in wild-type (+/+) and PC1/3-Null (-/-) mice. Neurointermediate lobe pituitaries from four PC1/3-Null mice were analyzed by SELDI-TOF MS using a $0.1 \mathrm{M} \mathrm{HCl}$ buffer, the same as for mice in Fig. 2. Ion peaks of 1009, 1031, 1084 and 1105 daltons were identified and correspond to oxytocin, oxytocin $+\mathrm{Na}^{+}, \mathrm{AVP}$ and $\mathrm{AVP}+\mathrm{Na}^{+}$.

portion of pro-vasopressin that is glycosylated, and thus its mass will be determined by the amount and type of glycosylation. The expected mass of glycopeptide is 4349 daltons without glycosylation. In a recent study of rat vasopressin, glycosylated glycopeptide was determined by mass spectrometry to be 5494 daltons (Jimenez et al. 1997). In the present study, two peptides have been found in the range between 5000 and 6000 daltons. 
Table 1 SELDI-TOF analysis of relative oxytocin and AVP amounts. The mean \pm S.E.M. was reported for the PC2-Null, heterozygote and wild-type groups $(n=3)$

\begin{tabular}{|c|c|c|c|c|c|}
\hline & PC2 & & & PC1 & \\
\hline & $-1-\mathrm{PC} 2$ & $-/+\mathrm{PC} 2$ & +/+ PC2 & $-/-P C 1$ & $+/+\mathrm{PC} 1$ \\
\hline$M / Z$ & & & & & \\
\hline 1009 & $4 \cdot 5 \pm 3$ & $1.04 \pm 1$ & $0.24 \pm 0.03$ & $5 \pm 3$ & $2 \pm 0.6$ \\
\hline 1030 & $16 \cdot 6 \pm 6$ & $14 \cdot 8 \pm 8$ & $5 \pm 1.5$ & $47 \pm 11$ & $24 \pm 14$ \\
\hline 1084 & $319 \pm 58$ & $321 \pm 16$ & $196 \pm 75$ & $319 \pm 70$ & $219 \pm 72$ \\
\hline 1105 & $124 \pm 23$ & $129 \pm 7$ & $61 \pm 24$ & $83 \pm 21$ & $42 \pm 17$ \\
\hline
\end{tabular}

The data represent the average integrated peak area for the peptide and its sodium adduct for each ion peak.

However, neither ion peak has been positively identified as glycopeptide using $\mathrm{C}$-terminal sequencing.

\section{Discussion}

The processing of prohormones to active hormones is a complex event that requires the coordinated action of specific processing enzymes such as PG1/3 and PC2, carboxypeptidase $\mathrm{E}$ and peptidylglycine $\alpha$-amidating monooxygenase (PAM) (Zhou et al. 1999). Some prior studies on prohormone processing have concentrated on the use of synthetic peptides and purified processing enzymes for in vitro analysis (Cawley et al. 1996, Coates \& Birch 1998, Olsen et al. 1998). Other studies have used over-expression of substrate and enzyme in a cell system (Rouille et al. 1992, Cool et al. 1996, Wang et al. 1998, Min et al. 1999). The drawback to these methods is the lack of regulation and biological relevance as found in vivo. For example, endogenous inhibitors and activators of processing enzymes may be present in intact cells, but absent from purified preparations. Over-expression of substrate and enzyme also lacks physiological relevance. In order to study the actual activity of prohormone processing enzymes, mice have been genetically altered, i.e. one of the processing enzymes (PC1/3 or PC2) has been 'knocked out'. The results presented here show the effects of deleting the PC2 processing enzyme on the formation of oxytocin/ AVP. The implication from this data is that PC2 is not required for processing pro-oxytocin or pro-vasopressin. Analyses of PC1/3-Null mice (Zhu et al. 2002a,b) were studied to further assess the role of $\mathrm{PCl} / 3$ in prooxytocin or pro-vasopressin processing and it was shown that $\mathrm{PC} 1 / 3$ is also not required for pro-oxytocin or pro-vasopressin processing. We interpret these results to show that both $\mathrm{PC} 1 / 3$ and $\mathrm{PC} 2$ can process the precursors, and if one is 'knocked out', the other enzyme 'takes over'. Proof of this will require double null mice.

For this paper, a relatively new, yet powerful, mass spectrometry technique was used to identify and quantitate the smaller peptide hormones, oxytocin and
AVP. SELDI-TOF MS was able to resolve oxytocin and AVP in extracts of neurointermediate lobe pituitary glands from all the mice analyzed. The results clearly show that pro-oxytocin and pro-vasopressin were correctly processed in the PC2-Null, heterozygote and wild-type mice. Furthermore, quantitative analysis revealed that there was no difference in the amount of AVP or oxytocin present in these mice. This is consistent with the results of Coates and Birch (1998) who concluded, based on studies with purified $\mathrm{PC} 1 / 3$, that PG1/3 enzyme was capable of (although not necessarily required for) processing pro-vasopressin to AVP. However in our study, when PC1/3-Null mice were analyzed for oxytocin and AVP, it appeared that both AVP and oxytocin were still present. These results can be compared with clinical conditions in which the lack of pro-oxytocin or pro-vasopressin processing is attributed to the putative absence of active PC2, for example in Prader-Willi syndrome and Wolfram's syndrome. The main hypothesis to explain these processing differences is that an enzyme redundancy may exist in mice, i.e. both $\mathrm{PC} 1 / 3$ or PC2 are capable of processing mouse pro-oxytocin and pro-vasopressin.

If $\mathrm{PC} 1 / 3$ or $\mathrm{PC} 2$ are both capable of processing pro-oxytocin and pro-vasopressin, then why are they not processed in humans with Prader-Willi syndrome or Wolfram's syndrome? The most plausible explanation is substrate specificity. That is, structural differences at or near the cleavage site in pro-oxytocin or pro-vasopressin would dictate the processing by a particular enzyme. When the amino acid sequences for mouse and human pro-oxytocin or pro-vasopressin were compared, the homology was $100 \%$ at the actual cleavage site, i.e. GKRA (Fig. 6). The region in mouse pro-oxytocin beginning with PLGG on the N-terminal side of the paired basic residue cleavage site represents a $\beta$-turn (see Fig. 6). The region on the C-terminal side of the paired basic residues with the sequence KRAVLDLDVR, represents an $\alpha$-helix (Brakch 2000). Both secondary structures appear to be required for proper processing (Fig. 6). However, for both human pro-vasopressin and pro-oxytocin, there are significant amino acid differences 


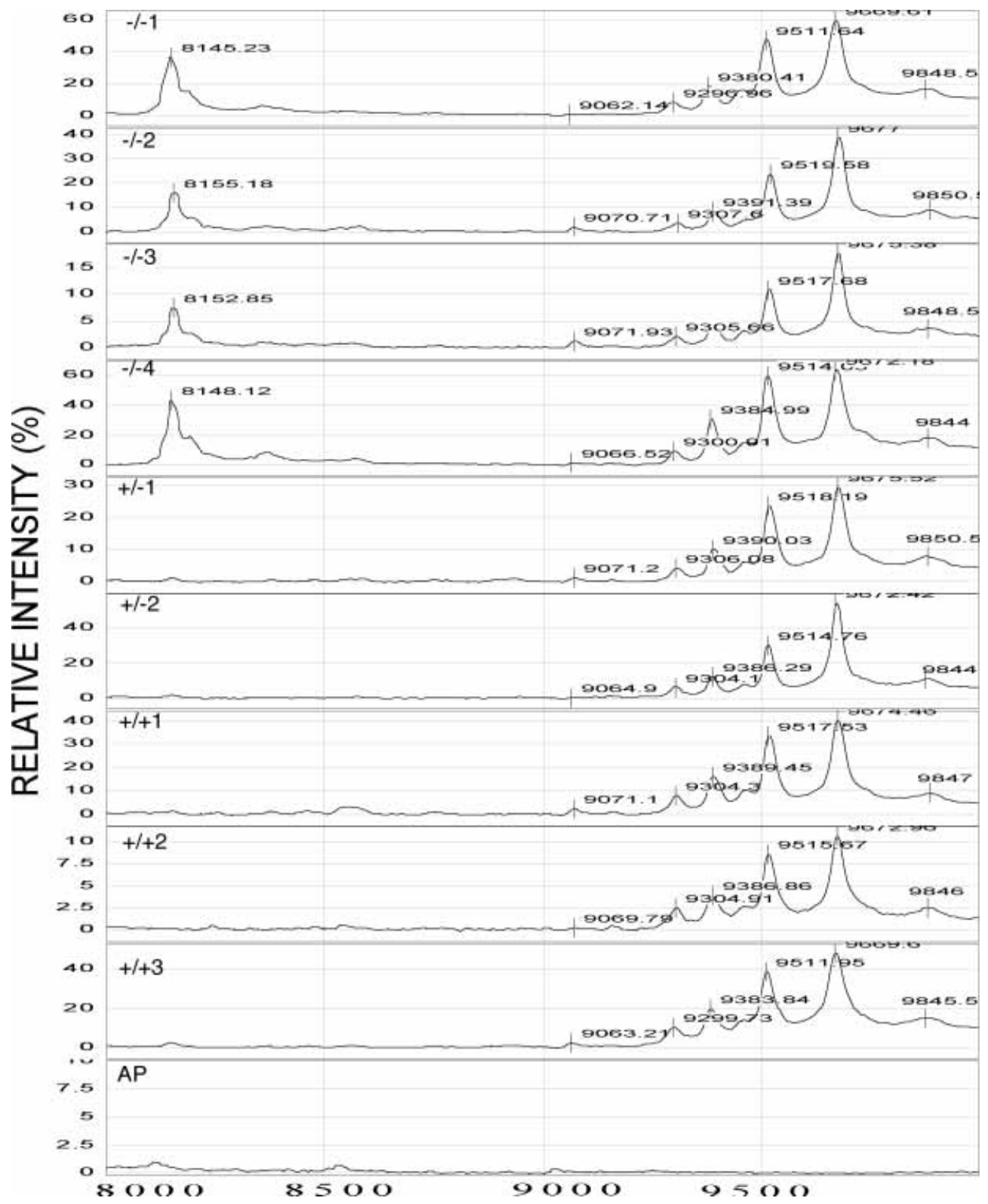

Figure 4 SELDI-TOF MS profiles of ion peaks in the 8000 to 10000 dalton range from PC2-Null $(-/-)$, heterozygote $(+/-)$ and normal mice $(+/+)$. Neurointermediate lobe pituitary lysates were analyzed using a WCX2 ProteinChip. The relative intensity (\%) was plotted for each peptide/protein (mass/charge, M/Z). A peak at $\sim 8100$ daltons was identified in the PC2-Null mice, but not in the heterozygote or wild-type mice. The bottom profile resulted from SELDI-TOF MS analysis of an anterior pituitary (AP) from a normal $(+/+)$ mouse.

in the same position within this $\alpha$-helix region, only 2 amino acids from the $\mathrm{C}$-terminus of the processing site. In human pro-vasopressin, a Met-residue replaces the Ile-residue (Fig. 6A). In human pro-oxytocin, Ala-Pro replaces Val-Leu, in this position (Fig. 6B). In both cases, a more hydrophobic amino acid is present in the mouse sequence compared with the human. The presence of the proline residue in human oxytocin is especially important for breaking the $\alpha$-helix structure. In addition, a Leu-residue is substituted for a Met-residue at the +5 position in human pro-vasopressin, and a Val-residue is substituted for a Met-residue at the +7 amino acid position in human pro-oxytocin (Fig. 6A and B). In a recent article on oxytocin processing by $\mathrm{PC} 1 / 3$, 
Table 2 Expected versus observed ion peaks for neurophysin I

\begin{tabular}{|c|c|c|c|c|}
\hline & Neurophysin I & & & \\
\hline & Expected daltons & Observed daltons & $\Delta$ Mass & Amino acid removed \\
\hline Sequence & & & & \\
\hline - DPACDPESAFSER ${ }^{*}$ & 9677 & $9676 \cdot 34$ & - & - \\
\hline - DPACDPESAFSE & 9521 & 9519.62 & $156 \cdot 72$ & $\mathrm{R}$ \\
\hline - DPACDPESAFS & 9392 & 9389.95 & $129 \cdot 67$ & $E$ \\
\hline - DPACDPESAF & 9305 & $9304 \cdot 10$ & 85.85 & $\mathrm{~S}$ \\
\hline - DPACDPESA & 9157 & $9158 \cdot 33$ & $145 \cdot 77$ & $\mathrm{~F}$ \\
\hline - DPACDPES & 9086 & $9086 \cdot 26$ & $72 \cdot 07$ & $A$ \\
\hline - DPACDPE & 9000 & $9000 \cdot 10$ & $86 \cdot 16$ & $S$ \\
\hline
\end{tabular}

${ }^{*}$ Only the last 13 amino acids of neurophysin I are shown. All ion peaks are in daltons and represent the monoisotopic single charge form of the peptide. The single amino acid code is used for the amino acid sequence. Expected indicates expected ion peak and Observed indicates observed ion peak. The amino acid column indicates the amino acid removed from the C-Terminus.

substitution of an alanine or valine residue on the C-terminal of the KR cleavage site with an amino acid that would block either the $\beta$-turn or the $\alpha$-helix caused disruption of the processing of pro-oxytocin by $\mathrm{PCl} / 3$ (Brakch 2000). Thus, the case for processing redundancy by PC1/3 or PC2 in mice based on structural specificity is strongly supported by the data presented here compared with the findings for Prader-Willi syndrome and Wolfram's syndrome. That is, amino acid differences in the human pro-oxytocin or provasopressin sequence near the processing site cause fundamental changes in the secondary structure that is required for correct processing.

An alternative hypothesis is that a third enzyme exists that is specific for processing pro-vasopressin and pro-oxytocin in mice but not in humans. This is supported by our results in which the processing of other prohormones, i.e. pro-opiomelanocortin, was perturbed in the PC2-Null mice, while pro-oxytocin and pro-vasopressin were processed normally. For this to occur, the enzyme would have to be expressed only in the posterior pituitary. Recent evidence suggests that another enzyme capable of processing pro-vasopressin and pro-oxytocin may be present in the paraventricular nucleus, PC5, although it was only observed in vasopressinergic but not oxyocinergic neurons (Dong et al. 1997).

Of further interest were the results obtained from the oxytocin-Null mice, since this is also the first analysis of the oxytocin-Null mouse by mass spectrometry. As was expected, oxytocin was not present in the neurointermediate lobe pituitary. However, of note was that two of the ion peaks in the neurophysin range, 9389 and 9519 daltons, were also missing, suggesting that these are neurophysin I-related peptides derived from the pro-oxytocin molecule. This is significant, because the lack of two of the peaks associated with neurophysin I further confirmed the sequence data. Another observation of note is that in virtually every neurointermediate lobe sample examined using SELDI-TOF MS, a series of 3-4 ion peaks in this region prevail, regardless of the preparation or use of proteolysis inhibitors. The observation of multiple peaks for the neurophysins is consistent with reports by others using MALDI-TOF MS to study rat AVP-related peptides and may represent the natural state of the peptides in this tissue (Jimenez et al. 1997).

Table 3 Expected versus observed ion peaks for neurophysin II

\begin{tabular}{|c|c|c|c|c|}
\hline & \multicolumn{2}{|l|}{ Neurophysin II } & \multirow[b]{2}{*}{$\Delta$ Mass } & \multirow[b]{2}{*}{ Amino acid removed } \\
\hline & Expected daltons & Observed daltons & & \\
\hline \multicolumn{5}{|l|}{ Sequence } \\
\hline -VAEPECHDGFFRLT* & 9679 & $9676 \cdot 34$ & - & - \\
\hline -VAEPECHDGFFRL & 9585 & 9585.00 & $91 \cdot 34$ & $\mathrm{~T}$ \\
\hline -VAEPECHDGFFR & 9473 & $9473 \cdot 20$ & $111 \cdot 8$ & $\mathrm{~L}$ \\
\hline -VAEPECHDGFF & 9315 & $9315 \cdot 81$ & $158 \cdot 39$ & $\mathrm{R}$ \\
\hline -VAEPECHDGF & 9168 & $9166 \cdot 16$ & 148.65 & $\mathrm{~F}$ \\
\hline
\end{tabular}

${ }^{*}$ Only the last 13 amino acids of neurophysin II are shown. All ion peaks are in daltons and represent the monoisotopic, single charge form of the peptide. Expected indicates expected ion peak and Observed indicates observed ion peak. The amino acid column indicates the amino acid removed from the C-terminus. 


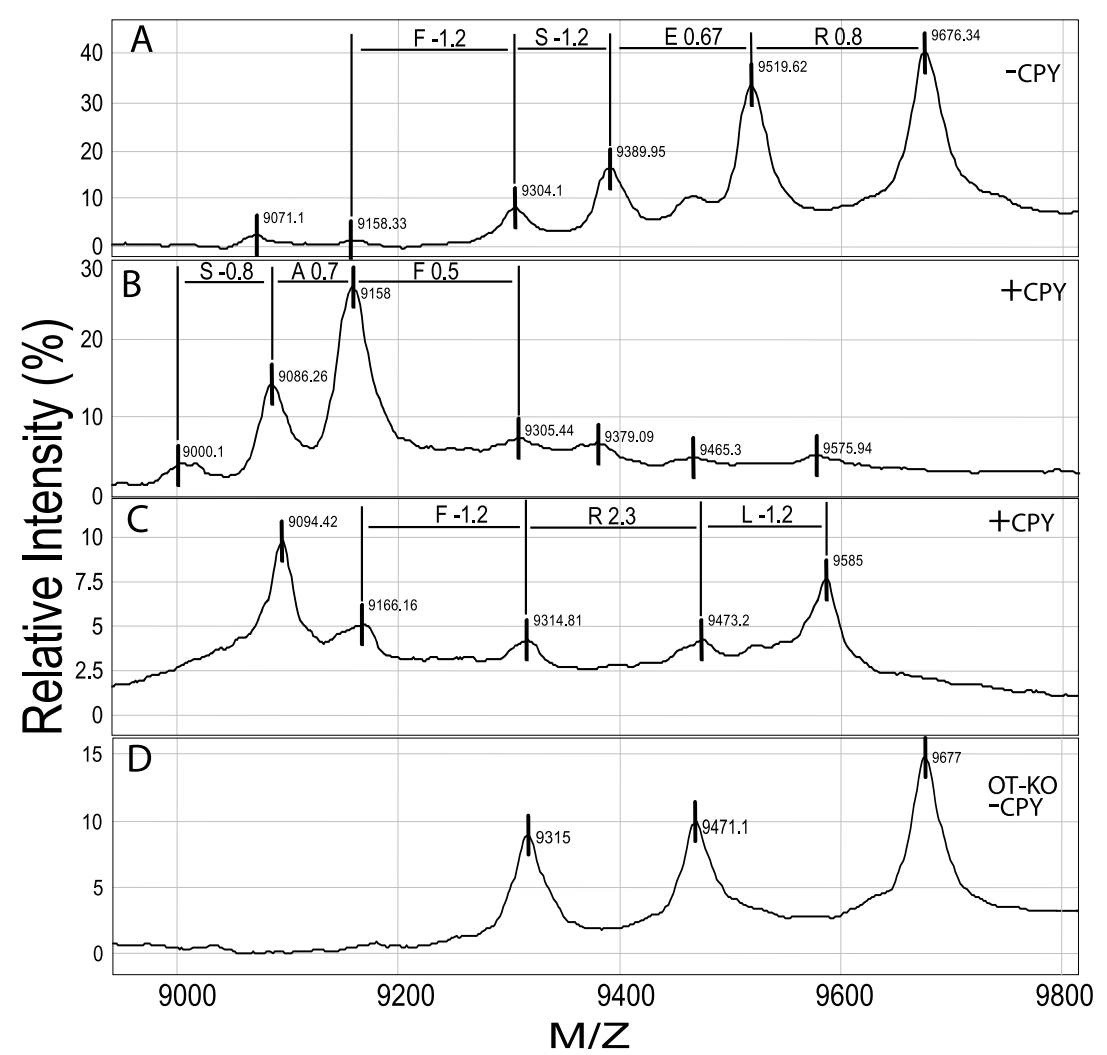

Figure 5 On-chip C-terminal sequence analysis of peptides in the 9000 to10 000 dalton range from PC2-Null mice. Both neurophysin I and neurophysin II have a predicted mass of $\sim 9677$ daltons. Naturally occurring ion peaks present before CPY treatment were 9676, 9519, 9389 and 9304 daltons (-CPY, A). Additional ion peaks characteristic of neurophysin I (+CPY, B; Table 2) and neurophysin II (+CPY, C; Table 3) were observed after treatment of the ProteinChips with CPY. Neurointermediate lobe pituitaries from oxytocin-Null mice were also analyzed (OT-KO -CPY; D) and were found to contain only three peaks characteristic of neurophysin II. Noticeably absent were the 9519 and 9389 peaks of neurophysin I. The single amino acid code is shown above the lines between peaks. The difference in daltons between the exact amino acid mass and the observed amino acid mass for each sequenced amino acid is also shown, i.e. R0.8, E0.67, etc.

Mass spectrometry represents a major advancement over RIAs and SDS-PAGE Western blots for analyzing peptide hormones in tissues such as the pituitary. The major advantages of mass spectrometry are: (1) the ability to analyze peptides that are too small to be resolved by SDS-PAGE, (2) the ability to quantitate the amount of the peptide and (3) the ability to confirm a peptide by its mass. Mass spectrometry, especially SELDI-TOF MS and MALDI-TOF MS, are excellent for analyzing peptides in the range between 500 and 10000 daltons. Another advantage of mass spectrometry is its level of sensitivity. For the Ciphergen SELDI-TOF MS used in this study, sensitivity in the attomole range has been reported (Hutchens 1993, Merchant \& Weinberger 2000). Another benefit of mass spectrometry compared with RIAs is the knowledge of the exact mass of the peptide being analyzed. The ability to quantitiate the peptides is similar to that of an RIA in that both require a standard curve with the known peptide. As used here, SELDI-TOF MS represents a new step in mass spectrometric peptide analysis by allowing 'onchip' peptide concentration and selective retentionbased purification (Hutchens 1993, Merchant \& Weinberger 2000). For traditional MALDI-TOF MS systems, the peptides must be desalted and peptides/ proteins purified prior to spotting on the metal MALDI targets. While similar results can be obtained, the on-chip method provides a much faster method for preparing the samples. The resolution and sensitivity is more than adequate for comparative analysis of peptides and proteins in a wide range of samples, e.g. tissues from knockout-animals, cells in culture, or synthetic peptides. 
A.
$\operatorname{AVP}(1,084 \mathrm{~d})$
$\downarrow+2+5$
$\operatorname{NPII}(9,679 d)$

hVP 1-CYFQNCPRGGKRAMSDLELRQCLPCGPGGKGRCFGPSICCADELGCFVGTAEALRCQEEN 60 Align CYFQNCPRGGKRA+SD+ELRQCLPCGPGGKGRCFGPSICCADELGCFVGTAEALRCQEEN mVP 1-CYFQNCPRGGKRAISDMELRQCLPCGPGGKGRCFGPSICCADELGCFVGTAEALRCQEEN 60 $\nabla \mathrm{GP}(4,349 \mathrm{~d})$

hVP YLPSPCQSGQKACGSGGRCAAFGVCCNDESCVTEPECREVFHRRARASDRSNATQLDGPA 120 Align YLPSPCQSGQK CGSGGRCAA G+CC+DESCV EPEC + F R RA + SNATQLDGPA mVP YLPSPCQSGQKPCGSGGRCAAVGI CC SDESCVAEPECHDGFFRLTRAREPSNATQLDGPA 120

hVP GALLLRLVQLAGAPEPFEPAQPDAY 145

Align ALLLRLVQLAG $E+A+P \quad Y$

mVP RALLLRLVQLAGTRESVDSAKPRVY 145

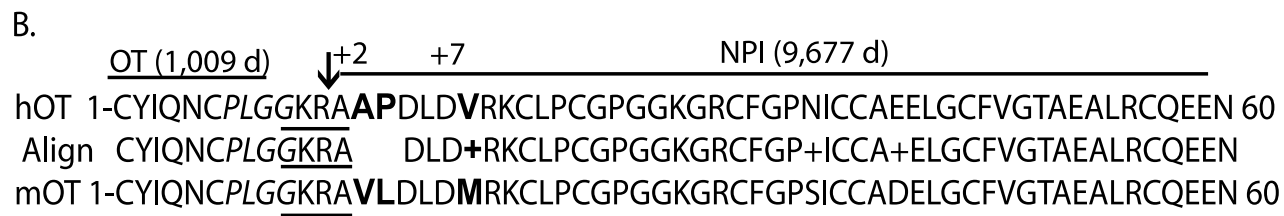

hOT YLPSPCQSGQKACGSGGRCAVLGLCCSPDGCHADPACDAEATFSQR 106

Align YLPSPCQSGQK CGSGGRCA G+CCSPDGC DPACD E+ FS+R mOT YLPSPCQSGQKPCGSGGRCAATGICCSPDGCRTDPACDPESAFSER 106

Figure 6 Blast amino acid sequence alignment for human and mouse pro-vasopressin $(A)$ and pro-oxytocin (B). The single-letter amino acid sequences for human and mouse pro-vasopressin (hVP or mVP respectively) and pro-oxytocin (hOT or mOT respectively), lacking the signal peptide region, were obtained from GenBank and analyzed by Blast sequence alignment (www.ncbi.nlm.nih.gov/blast/bl2 seq/wblast2.cgi). Accession numbers were: hOT \#AY082910; mOT \#AF483509; hVP \#NM000490; mVP \#NM009732. The amino acid sequence homology is displayed between the human and mouse sequence (Align). The homology was $>82 \%$ for pro-vasopressin (119/145 amino acids) and $>85 \%$ for pro-oxytocin (91/106 amino acids). Numbering before and after each line indicates the number of amino acid residues in that line. Spaces in the Align row indicate no homology, (+) indicates similar charge substitutions. The peptide hormones AVP, oxytocin (OT), neurophysin (NPI and NPII) and the glycopeptide region (GP), along with their predicted mass in daltons, e.g. $1084 \mathrm{~d}$, are indicated by the bar above their corresponding amino acid sequence. The arrows indicate the paired-basic residue prohormone cleavage site in the two prohormones. The arrowhead indicates the cleavage site between NPII and glycopeptide in pro-vasopressin. The GKRA cleavage site is underlined in each sequence. The PRGG conserved sequence is in italics and the $+2,+5$ and +7 amino acid changes are indicated in bold type.

Thus, the SELDI-TOF MS method presented here provides a unique and powerful way for the investigator to quickly analyze numerous and complex biological samples.

In conclusion, we have analyzed the neurointermediate lobe pituitary proteome from a prohormone processing enzyme null mouse using a specialized mass spectrometric technique, SELDI-TOF MS. The results clearly show that the two neurointermediate pituitary prohormones, pro-oxytocin and pro-vasopressin, do not require the activity of either PG1/3 or PC2, suggesting that the enzymes are redundant and one enzyme can process the precursor in the absence of the other. The results suggest that a proteomic examination of the spectra from a specific tissue from wild-type and null mice can yield useful information that can help identify and characterize the effect of an enzyme on prohormone processing. The proteomic analysis of endocrine and neuro-endocrine tissues and peptides is part of a multifaceted and comprehensive analysis of the endocrine system called 'endocrinomics', and techniques such as SELDI-TOF MS will play a unique and vital role in developing the endocrinomic profile of a cell, tissue or animal. 


\section{Acknowledgements}

The authors wish to thank Dr Mariana Morris (Wright State University, School of Medicine, Dayton, $\mathrm{OH}$, USA) for the generous donation of the oxytocin-Null mice for this study and Dr Sau-Mei Leung (Bruker Daltronics, Billerica, MA, USA) for the MALDI-TOF/ TOF analysis. This work was supported by grants from the Department of Defense (DAMD17-00-C-0020) and NIH (R01DK/NS58111) (to D R G). T G F is supported by NIH (R24 DA017298) Minority Institution Drug Abuse Research Program (MIDARP) and a Center of Clinical Research Excellence grant (U54 RR14616-01), both to Charles R Drew University of Medicine and Sciences. DFS is supported by NIH grants DK13914 and DK20595 and by the Howard Hughes Medical Institute. The authors declare that there is no conflict of interest that would prejudice the impartiality of this scientific work.

\section{References}

Brakch N, Rholam M, Simonetti M \& Cohen P 2000 Favourable side-chain orientation of cleavage site dibasic residues of prohormone in proteolytic processing by prohormone convertase 1/3. European Fournal of Biochemistry 267 1626-1632.

Cawley NX, Chen HC, Beinfeld MG \& Loh YP 1996 Specificity and kinetic studies on the cleavage of various prohromone monoand paired-basic residue sites by yeast aspartic protease 3. Fournal of Biological Chemistry 271 4168-4176.

Coates LC \& Birch NP 1998 Differential cleavage of pro-vasopressin by the major molecular forms of SPC3. Fournal of Neurochemistry $\mathbf{7 0}$ 1670-1678.

Cool DR \& DeBrosse D 2003 Extraction of oxytocin and arginine-vasopressin from serum and plasma for radioimmunoassay and surface enhanced laser desorption-ionization time of flight mass spectrometry. Fournal of Chromatography B 792 375-380.

Cool DR \& Hardiman A 2004 C-terminal sequencing of peptide hormones using carboxypeptidase $\mathrm{Y}$ and SELDI-TOF mass spectrometry. Biotechniques 36 32-34.

Cool DR, Louie DY \& Loh YP 1996 Yeast aspartic protease 3 (YAP3p) is sorted to secretory granules and activated to process pro-opiomelanocortin in PG12 cells. Endocrinology 137 5441-5446.

Darby NJ \& Smyth DG 1990 Endopeptidases and prohormone processing. Bioscience Reports 10 1-13.

Dong W, Seidel B, Marcinkiewicz M, Chretien, M, Seidah NG \& Day R 1997 Cellular localization of the prohormone convertases in the hypothalamic paraventricular and supraoptic nuclei: selective regulation of PC1 in corticotrophin-releasing hormone parvocellular neurons mediated by glucocorticoids. Fournal of Neuroscience 17 563-575.

Furuta M, Yano H, Zhou A, Rouille Y, Holst JJ, Carroll R, Ravazzola M, Orci L, Furuta H \& Steiner DF 1997 Defective prohormone processing and altered pancreatic islet morphology in mice lacking active SPC2. PNAS 94 6646-6651.

Gabreels BA, Swaab DF, Seidah NG, Van Duijnhoven HLP, Martens GJM \& Van Leeuwen FW 1994 Differential expression of the neuroendocrine polypeptide 7B2 in hypothalami of Prader-(Labhart)-Willi syndrome patients. Brain Research 657 281-293.
Gabreels BATF, Swaab DF, De Dleijn PV, Seidah NG, Van de Loo J-W, Van de Ven WJM, Martens GJM \& Van Leeuwen FW 1998 Attenuation of the polypeptide 7B2, prohormone convertase PC2, and vasopressin in the hypothalamus of some Prader-Willi patients: indications for a processing defect. Fournal of Clinical Endocrinology and Metabolism 83 591-599.

Hutchens TW \& Yip TT 1993 New desorption strategies for the mass spectrometric analysis of macromolecules. Rapid Communications in Mass Spectrometry 7 576-580.

Jimenez CR, Li KW, Dreisewerd K, Mansvelder HD, Brussard AB, Reinhold BB, Van Der Schors RC, Karas M, Hillenkamp F, Burbach JPH et al 1997 Pattern changes of pituitary peptides in rat after salt-loading as detected by means of direct, semiquantitative mass spectrophotmetric profiling. PNAS 94 9481-9486.

Lindberg I 1991 The new eukaryotic precursor processing proteinases. Molecular Endocrinology 5 1361-1365.

Loh YP 1988 Processing of pro-opiomelanocortin and other peptide precursors by unique, secretory vesicle enzymes. In Molecular Mechanisms in Secretion, pp 525-539. Eds NA Thorn, M Treiman \& $\mathrm{OH}$ Petersen. Copenhagen: Munksgaard.

Marck C 1988 'DNA Strider': a 'C' program for the fast analysis of DNA and protein sequences on the Apple Macintosh family of computers. Nucleic Acids Research 16 1829-1836.

Merchant M \& Weinberger SR 2000 Recent advancements in surface-enhanced laser desorption/ionization-time of flight-mass spectrometry. Electrophoresis 21 1164-1177.

Min L, Shuto Y, Somogyvári-Vigh A \& Arimura A 1999 Prohormone convertases 1 and 2 process proPACAP and generate matured, bioactive PACAP38 and PACAP27 in transfected rat pituitary GH4C1 cells. Neuroendocrinology 69 217-226.

Nishimori K, Young LJ, Guo Q Wang Z, Insel TR \& Matzuk MM 1996 Oxytocin is required for nursing but is not essential for parturition or reproductive behavior. PNAS 93 11699-11704.

Olsen V, Guruprasad K, Cawley N, Chen H, Blundell T \& Loh Y 1998 Cleavage efficiency of the novel aspartic protease yapsin 1 (Yap3p) enhanced for substrates with arginine residues flanking the P1 site: correlation with electronegative active-site pockets predicted by molecular modeling. Biochemistry 37 2768-2777.

Rouille Y, Spang A, Chauvet J \& Acher R 1992 Evidence for distinct dibasic processing endopeptidases with Lys-Arg and Arg-Arg specificities in neurohypophysial secretory granules. Biochemical and Biophysical Research Communications 183 128-137.

Seidah NG, Day R, Benjannet S, Rondeau N, Boudreault A, Reudelhuber T, Schafer MK-H, Watson SJ \& Chretien M 1992 The prohormone and proprotein processing enzymes PCl and PC2: structure, selective cleavage of mouse POMC and human renin at pairs of basic residues, cellular expression, tissue distribution, and mRNA regulation. NIDA Research Monograph 126 $132-150$.

Seidah NG, Day R, Marcinkiewics M \& Chretien M 1993 Mammalian paired basic amino acid convertases of prohormones and proproteins. Annals of the New York Academy of Sciences $\mathbf{6 8 0}$ 135-146.

Slominski A, Szczesniewski A \& Wortsman J 2000 Liquid chromatography-mass spectrometry detection of corticotropin-releasing hormone and proopiomelanocortin-derived peptides in human skin. Fournal of Clinical Endocrinology and Metabolism 85 3582-3588.

Steiner DF, Smeekens SP, Ohagi S \& Chan SJ 1992 The new enzymology of precursor processing endoproteases. Fournal of Biological Chemistry 267 23435-23438.

van Strien FJC, Jepersen S, van der Greef J, Jenks BG \& Roubos EW 1996 Identification of POMC processing products in single melanotrope cells by matrix assisted laser desorption/ionization mass spectrometry. FEBS Letters 379 165-170.

Swaab DF, Purba JS \& Hofman MA 1995 Alterations in the hypothalamic paraventricular nucleus and its oxytocin neurons 
(putative satiety cells) in Prader-Willi syndrome: a study of five cases. Fournal of Clinical Endocrinology and Metabolism $\mathbf{8 0}$ 573-579.

Wang W, Birch NP \& Beinfeld MC 1998 Prohormone convertase 1 (PC1) when expressed with pro cholecystokinin (pro CCK) in $\mathrm{L}$ cells performs three endoproteolytic cleavages which are observed in rat brain and in CCK-expressing endocrine cells in culture, including the production of glycine and arginine extended CCK8. Biochemical and Biophysical Research Communications 248 538-541.

Zhou A, Webb G, Zhu X \& Steiner DF 1999 Proteolytic processing in the secretory pathway. Fournal of Biological Chemistry 274 20745-20748.
Zhu X, Orci L, Carroll R, Norrbom C, Ravazzola M \& Steiner DF $2002 a$ Severe block in processing of proinsulin to insulin accompanied by elevation of des-64,65 proinsulin intermediates in islets of mice lacking prohormone convertase 1/3. PNAS 99 10299-10304

Zhu X, Zhou A, Dey A, Norrbom C, Carroll R, Zhang C, Laurent V, Lindberg I, Ugleholdt R, Holst J \& Steiner DF $2002 b$ Disruption of PC1/3 expression in mice causes dwarfism and multiple neuroendocrine peptide processing defects. PNAS 99 10293-10298.

Received 28 February 2005

Accepted 23 March 2005 